\title{
The formation of residual stresses in the bonding of automotive glass
}

\author{
Nikolay Nosov, Roman Grishin*, Vladimir Rodionov, and Roman Ladyagin \\ Samara state technical university, Russia
}

\begin{abstract}
This article discusses the formation of residual stresses during the assembly of automotive glass. The influence of various parameters of the adhesive bead on the spatial deviations of glasses during their installation is shown. Currently, the glass assembly technology involves filling the gap between the automotive glass and the car body with a glue roller. It has been established that the formation of residual stresses is influenced by the width and thickness of the glue roll, with a minimum width of the glue roll during drying, a discontinuity of the glue joint along the contour is possible, and with a maximum width, unacceptable residual deformations occur in the glass, which lead to cracks.
\end{abstract}

\section{Introduction}

When assembling automotive glasses, it is necessary to comply with several established requirements for the operation of the product. This is the tightness of the glass-body connection to protect from weathering, and the strength of the adhesive bond, which affects the spatial accuracy and rigidity of the entire structure [1].

As a rule, when connecting several media with different physical properties (or parameters) in technical systems (machines, apparatuses, etc.) there is a problem of sealing such connections. Various seals (adhesives) are used to seal detachable joints.

To take into account the effect of residual stresses in the glass on the accuracy of the installation, an analysis of the assembly process was carried out using real values of the gaps. In this case, due to the difference in the size of the gap along the perimeter of the glass, the glue roller will experience different deformation. Accordingly, the elastic recovery in the glue line will be uneven, the shrinkage during the polymerization of the glue will be uneven. All this leads to uneven technological residual stresses in the adhesive and glass.

\section{Contact model}

The design model of the assembly unit, which allows simulating the main features of glass embedding (gluing) into the car body, is presented in a generalized form in Fig. 1.

\footnotetext{
* Corresponding author: konferencia-isap@yandex.ru
} 
Using FEM, the calculation data the stress-strain state (SSS) of glass as an object included in a complex structure (assembly unit) were obtained and the rheological processes occurring in the adhesive bond during and after glass assembly were studied.

Initial data of the model: a fixed connection of a glass element 1 of a car with elements of a power set 2 (car body) is shown, which is carried out through an elastic adhesive bead 3 , made of polyurethane material (Terostat glue - 8590), and located below on the glass surface in the form layer of a deformable base.

In our case, the problem of determining stresses and deformations in a thin flat glass shell [2], which has a complex contour in plan and is based on an elastic-deformable base (adhesive bead), is solved numerically.

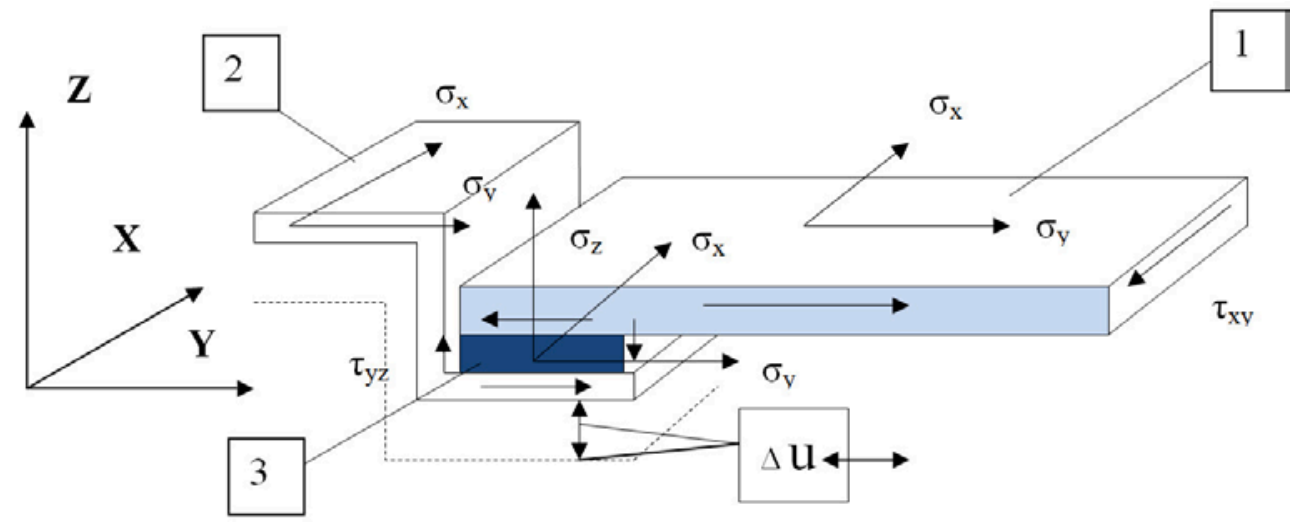

Fig. 1. Design model of glass assembly: 1 - glass, 2 - car body, 3 - adhesive bead

The influence of the geometrical parameters of the glue on the stress-strain state of the glass assembly during its operation has been proved. Based on the results of calculations obtained by the numerical method under torsional loading of the car body, it was proved that the value of residual stresses depends on the height and width of the adhesive bead and obeys the following regression relationships:

$$
\begin{aligned}
& f(b)=-0.0618 b^{2}+1.373 b+16.584 \\
& f(h)=-0.0405 h^{2}+0.0381 h+20.261
\end{aligned}
$$

where $f(b)$ and $f(h)$ are functions of the dependence of residual stresses on the width $b$ and height $h$ of the adhesive bead.

The obtained dependences make it possible to change the stress-strain state of the glass, and, accordingly, the residual stresses, using the stiffness parameter of the adhesive bead and the gaps obtained during dimensional analysis, as a deformable glass base that receives and transmits the load on the glass in the assembly and operation.

The calculation data allow us to conclude that it is possible to control the formation of the glass residual stress field by changing the geometric parameters of the adhesive in the assembly.

The model shown in Fig. 1 does not take into account the rheological behavior of the adhesive bead, but this is important because when assembling the glass, the adhesive undergoes changes and its properties are depend up by the height of the gap between the glass and the car body, the curing time, and the pressing force. In view of the fact that the glue affects the stress-strain state of glass $(1,2)$ it is necessary to develop a rheological model of the glue. 


\section{Unpolymerized adhesive model}

To determine the physical and mechanical properties of the adhesive bead used in glass assembly, a mathematical model of unpolymerized glue has been developed. In unpolymerized form, its behavior is described by an elastic-plastic rheological model [3]. The viscous component can be ignored, since the Assembly force is applied at a low speed, and the loading can be considered quasi-static [4].

The elastic - plastic body model is described by the equation:

$$
s_{i j}=2 G\left(f_{1}\left(\varepsilon_{u}\right) \ni_{i j}-\int_{0}^{t} R(t-\tau) f_{2}\left(\varepsilon_{u}\right) \ni_{i j} d \tau, \sigma=3 K \cdot \varepsilon\right.
$$

where $S_{i j}, \ni_{i j}, \sigma, \varepsilon$ - deviatorial and spherical parts of stress and strain tensors; $R(t-\tau)$ - relaxation kernel; $f_{1}\left(\varepsilon_{и}\right)$ - Ilyushin plasticity function; $f_{2}\left(\varepsilon_{и}\right)$ - universal nonlinear creep function; $\varepsilon_{H}$ - strain intensity; $G$ - shear modulus; $K$ - bulk modulus.

Ilyushin plasticity function:

$$
\begin{gathered}
f_{1}\left(\varepsilon_{u}\right)=1-\omega_{1}\left(\varepsilon_{u}\right) \\
\text { where } \omega_{1}\left(\varepsilon_{u}\right)=\left\{\begin{array}{lr}
0, & \varepsilon_{u}<\varepsilon_{T o} \\
A_{1}\left(1-\frac{\varepsilon_{T o}}{\varepsilon_{u}}\right)^{\alpha}, & \varepsilon_{u} \geq \varepsilon_{T o}
\end{array}\right.
\end{gathered}
$$

where $\mathcal{E}_{T o}$ - strain intensity corresponding to the yield strength.

To determine the elastic modulus of the adhesive, the loading curve was differentiated (Fig. 2).

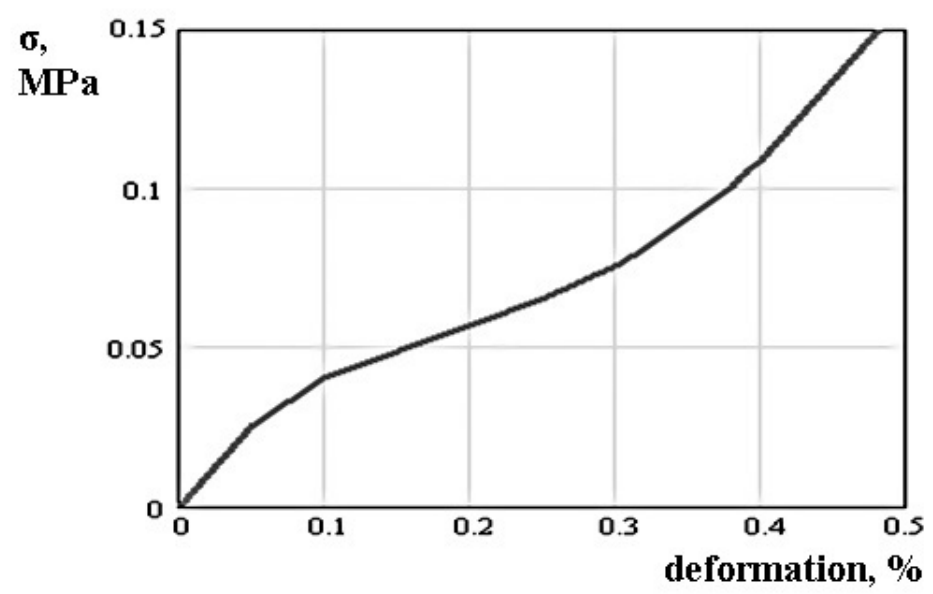

Fig. 2. Loading curve

The set of values of the indicator $\alpha$ and the coefficient $A_{1}$ from the experimental loading curve at the measurement points was determined. After averaging, the following values were obtained: $A_{1}=0,18 ; \alpha=3,24$. The universal creep function is determined by the formula, which includes the second derivative of stress with respect to deformation A.K. Grigorieva [5] 


$$
f_{2}\left(\varepsilon_{u}\right)=-\frac{1}{E} \frac{\Delta^{2} \sigma}{\Delta \varepsilon^{2}}
$$

After regression analysis of the experimental curve, the strain-force for unpolymerized adhesive is determined (Fig.2):

$$
f_{2}\left(\varepsilon_{u}\right)=-\frac{1}{E}\left(1,492+2,292 \cdot t-23,664 \cdot t^{2}\right)
$$

where $\varepsilon_{u}=\varepsilon_{0} t$ - change in strain over time.

To describe the relaxation processes, the Rzhanitsyn relaxation kernel was used in the form [6]:

$$
R(t)=A \cdot e^{-\beta \cdot t} \cdot t^{\alpha_{1}-1},(\beta<0,0<\alpha<1)
$$

The experimental $\sigma(t)$ curve was transformed into $\sigma(t) / \sigma_{o}(t)$, where $\sigma_{o}(t)$ is the initial stress. Accordingly, taking into account the values of the difference in forces in the adhesive bead for each side, the values of the difference in residual deformations, stresses and the gap between the glass and the car body were obtained.

Taking into account the developed model of unpolymerized adhesive, the residual stresses in the glass after assembly were calculated. In Fig. 3 shows the difference in the magnitude of residual stresses along the edge of the glass for the basic (old) and design (new) assembly diagram, arising from the difference in efforts during assembly and the difference in glue shrinkage, taking into account the deviation of the geometry of technological gaskets.

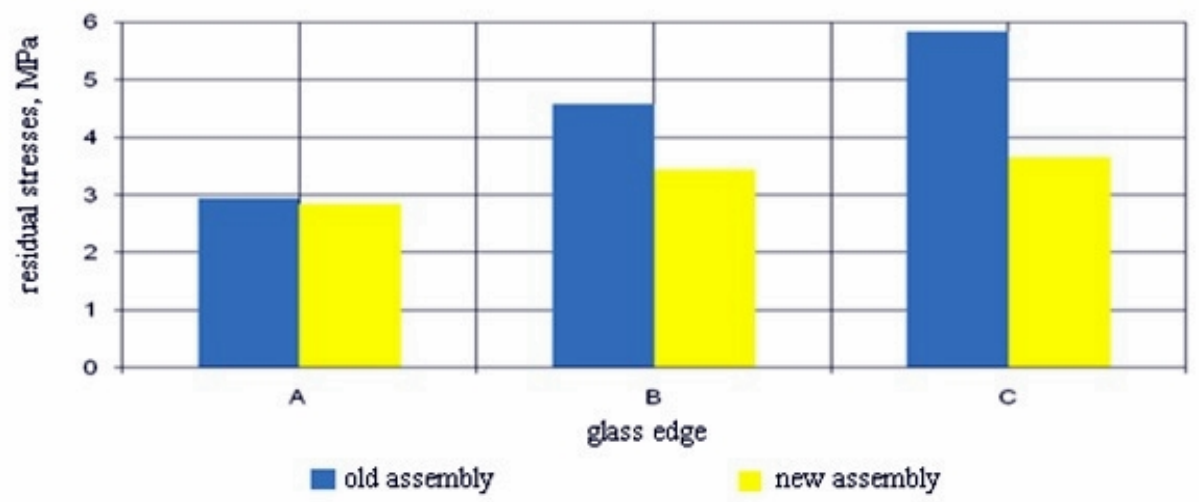

Fig. 3. Distribution of residual stresses in glass edges after assembly. A - lower edge of glass, B upper edge of glass, $\mathrm{C}$ - side edge of glass.

Research has been carried out on the adhesive assembly of glasses, taking into account two options for locating glasses on gaskets (using a guide base - 2 gaskets and using an installation base - 3 gaskets) and the effect of an error in the thickness of technological gaskets on the spatial arrangement of glass.

After processing the data of dimensional analysis using the probabilistic method, the advantage of the proposed glass location diagram in the assembly ( 3 gaskets) was proved. It was found that in the used assembly diagram, the reference points when basing are located on the same line. In the proposed version, three reference points are spaced along the perimeter of the glass, which changed the layout of the gaskets, which reduced the spatial 
skew of the glass. In this case, technological gaskets can be applied using the selective assembly method.

When changing the basing scheme, the scattering field of the gap between the glass and the car body decreases. Dimensional analysis showed that the largest size obtained by measuring the geometric model after the changes made is $5.487 \mathrm{~mm}$, then the upper deviation is: $\mathrm{ES}_{\text {true }}=5.487-4.9=0.587 \mathrm{~mm}$. The final size of the gap is ensured after polymerization of the adhesive.

With the basic version of the glass assembly and the nominal size of the gaskets located along the lower edge of the glass, residual stresses arise in the glass after assembly $\sigma_{\text {res.max }}=$ 4.5 MPa. With lateral skewing of the glass arising from the deviation of the geometry of the gaskets residual stresses arise in the glass $\sigma_{\text {res.max }}=5.83 \mathrm{MPa}$. In the case of frontal skewing arising from the difference in force and the absence of technological gaskets along the upper edge of the glass, residual stresses arise in the glass $\sigma_{\text {res.max }}=5.57 \mathrm{MPa}$.

It is known [7] that the glass supplied to AvtoVAZ contains tensile technological residual stresses $\sigma_{\text {initial }}=10 \mathrm{MPa}$. Thus, in the assembly, these stresses are superimposed on those existing in the glass and the uniformity of the residual stress field is disturbed, causing excessive stress on the glass after assembly.

When using the design version of the Assembly with a new basing scheme, the value of residual stresses in the glass is reduced by $\approx 40 \%$. At the same time, with the nominal size of the gaskets arranged in a three-support scheme, residual stresses arise in the glass after assembly $\sigma_{\text {res.max }}=2.83 \mathrm{MPa}$. In the case of lateral skewing of the glass, arising from the deviation of the geometry of the gaskets, residual stresses arise in the glass $\sigma_{\text {res.max }}=3.43$ $\mathrm{MPa}$. With frontal skewing arising from the difference in force: $\sigma_{\text {res.max }}=3.65 \mathrm{MPa}$.

\section{Conclusion}

Thus, the research allowed us to conclude that the geometric parameters of the adhesive bead - its height and width-affect the redistribution of residual stresses in the glass during the car body operation. Based on the data obtained in the study of rheological processes occurring in the adhesive bead after glass assembly, the influence of the accuracy of the geometry of the car body elements on the formation of residual stresses in the adhesive bead and glass after assembly is proved. In addition, it was found that the following factors affect the quality of glass assembly and its strength indicators: the glass basing scheme, the assembly method, the tolerance for the deviation of the car body geometry.

\section{References}

1. K.Z. Galimov, V.N. Paimushin, Theory of shells of complex geometry (Kazan: Publishing house Kazan, University, 1985)

2. N.M. Pavlushkin, Glass (Moscow, "Stroyizdat", 1973)

3. I.A. Birger, Y.G. Panovko, Strength. Stability. Oscillations (M .: "Mechanical engineering", 1968)

4. M.S. Kornishin, V.N. Paimushin, V.F. Snigirev, Computational geometry in problems of shell mechanics (Moscow: Nauka, 1989)

5. V.N. Paimushin, Glass shells. Calculation of the stress-strain state (Moscow: Mashinostroenie, 1993)

6. A.S. Freidin, Strength and durability of adhesive joints (Moscow: Chemistry, 1971)

7. V.K. Shtrobel, Modern automobile body (M: Translated from German by N.A. Yunikova. "Mechanical Engineering", 1984) 